\title{
Lista preliminar das espécies da macrofauna de fundos inconsolidados da Baía de Guaratuba, Paraná, Brasil
}

\author{
Arno Blankensteyn ${ }^{1}$ \\ Ronny Scheffler de Moura ${ }^{2}$
}

\begin{abstract}
Preliminary list of softbottom benthic macrofauna from Guaratuba Bay, Paraná, Brazil. A qualitative survey of benthic macrofauna was carried out in the Guaratuba Bay, Paraná, Brazil. Twenty nine stations were sampled from April to July, 1996, including sublitoral and intertidal zones resulting in a preliminary list with 69 taxa. From this total, only $31,9 \%$ (22 taxa) were previously registered to the area. KEY WORDS. Guaratuba Bay, benthic macrofauna, qualitative study, soft bottom
\end{abstract}

A macrofauna bentônica é ainda pouco conhecida para o litoral brasileiro. Recentes e abrangentes estudos de sistemática de invertebrados de fundos inconsolidados são escassos, devido principalmente à falta de formação de especialistas e à falta de recursos para as amostragens oceanográficas. Os estudos da variabilidade espacial e temporal das comunidades bentônicas são ainda mais raros.

Historicamente, os estudos de levantamentos faunísticos são documentos importantes para o conhecimento e monitoramento de áreas de relevante interesse, como é o caso dos estuários. TOMMASI (1967; 1970), BEMVENUTI et al. (1978), CAPITOLI et al. (1978), LANA (1986b) BEMVENUTI et al. (1992) forneceram informações sobre a macrofauna bentônica de importantes sistemas estuarinos da costa sul-sudeste do Brasil.

Em relação aos estudos realizados para o litoral do Paraná, pode-se dizer que os trabalhos mais abrangentes sobre a macrofauna bentônica, e também os mais recentes, referem-se à Baía de Paranaguá (LANA 1986a). Entre os estudos desenvolvidos na Baía de Guaratuba, citam-se Marcus (1941), SAwaya (1945), GoFFERJÉ (1950), BJORNBERG (1953), JAKOBI (1953, 1955), LOYOLA E SILVA (1959, 1992), Lana (1986b), Müller \& LaNa (1986), Melo et al. (1989). Morretes (1949) foi provavelmente quem produziu o primeiro catálogo de moluscos do Brasil, sendo que nesse trabalho pioneiro já contava com material proveniente da região de Guaratuba (Therezinha M. Absher, com. pes.).

O objetivo do presente trabalho é listar as espécies da macrofauna bentônica de fundos inconsolidados da Baía de Guaratuba, Paraná.

1) Departamento de Ecologia e Zoologia, Universidade Federal de Santa Catarina. Campus Trindade, Caixa Postal 476, 88040-900 Florianópolis, Santa Catarina, Brasil.

2) Curso de Ciências Biológicas, Universidade Federal do Paraná. Centro Politécnico, Jardim das Américas, Caixa Postal 19020, 81531-980 Curitiba, Paraná, Brasil.

Revta bras. Zool. 19 (3): 715 - 721, 2002 


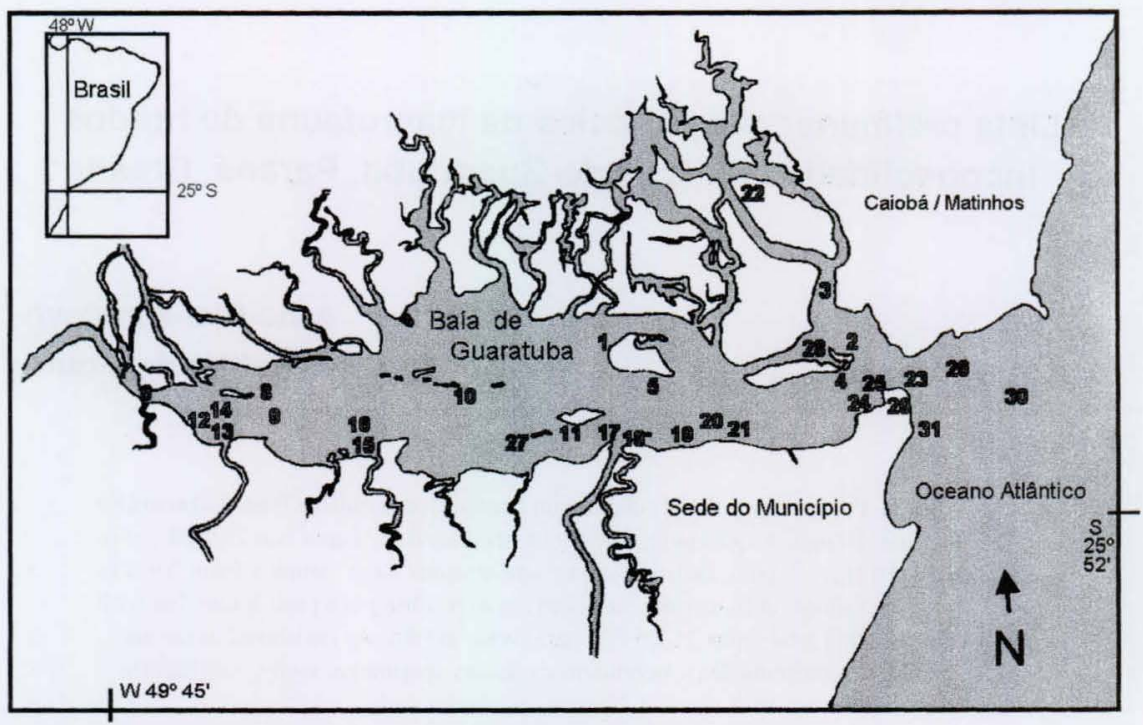

Figura 01. Mapa da área de estudo com indicação dos pontos de coleta

Fig. 1. Mapa da Baia de Guaratuba com as localizações das estações de coleta, numeradas de 1 a 29.

\section{MATERIAL E MÉTODOS}

A Baía de Guaratuba está situada entre as latitudes $25^{\circ} 50^{\prime}$ e $25^{\circ} 55^{\prime} \mathrm{S}$ e longitudes 4830' e 4850' W (Fig. 1).

O estuário apresenta orientação leste-oeste com aproximadamente $16 \mathrm{Km}$ de comprimento e largura de $3 \mathrm{Km}$. A largura máxima pode chegar a $10 \mathrm{Km}$ quando consideradas as planícies de marés (PETROBRÁs 1994). A abertura do estuário para o mar aberto tem largura de 700 m. MAACK (1968) e BigARELLA $(1946,1978)$ forneceram informações gerais sobre a geologia e ecologia da região litorânea do Estado do Paraná.

Foram realizadas coletas qualitativas no período de 26 de abril a 20 de junho de 1996, quando procurou-se amostrar os diversos setores do estuário. Com exceção dos manguezais, os demais ambientes entre-marés vegetados do estuário como as marismas de Spartina alterniflora Loisel, 1890 e os bancos de Crinum sp. foram investigados.

A tabela I apresenta algumas informações e características das estações de coleta (data e método de coleta, local e profundidade).

Para as amostras em fundos sublitorais utilizou-se os métodos de arrasto com rede de porta e amostrador de fundo tipo Van Veen. Na região entre-marés realizouse amostragem manual, com pás. O material biológico proveniente das coletas foi triado sobre peneiras de $1 \mathrm{~mm}$, fixado em formalina $4 \%$ e posteriormente conservado em álcool a $70 \%$.

Indivíduos juvenis e fragmentos não foram identificados a nível específico e alguns foram mantidos a nível de grandes grupos. 
Tabela I. Sumário das informações e características das estações de coleta na Baía de Guaratuba, Paraná.

\begin{tabular}{|c|c|c|c|c|}
\hline Estaçāo & Data & Local & Método & Profundidade \\
\hline 1 & $26 / 04 / 96$ & Ao lado da llha do Araçá & Arrasto de porta e Van Veen & 8 \\
\hline 2 & $26 / 04 / 96$ & Parte abrigada da liha da Sepultura (remanso) & Van Veen & 4 \\
\hline 3 & $26 / 04 / 96$ & Em frente ao iate Clube Caiobá & Arrasto de porta e Van Veen & 4 \\
\hline 4 & $26 / 04 / 96$ & Entre as Ilhas da Pescaria e da Sepultura & Van Veen & 4 \\
\hline 5 & $26 / 04 / 96$ & Atrás da llha do Araça & Arrasto de porta Van Veen & 5 \\
\hline 6 & $10 / 05 / 96$ & Desembocadura do Rio São Joãozinho & Van Veen & 7 \\
\hline 7 & $10 / 05 / 96$ & Desembocadura do Rio Chapéu & Van Veen & 1 \\
\hline 8 & $10 / 05 / 96$ & Ponta da Coroa Grande (baixio) & Van Veen & 1 \\
\hline 9 & $10 / 05 / 96$ & Ponta da Coroa Grande (canal) & Van Veen & 3 \\
\hline 10 & $10 / 05 / 96$ & Canal do Capim do Meio & Van Veen & 4 \\
\hline 11 & $10 / 05 / 96$ & Ilha das Garças & Van Veen & 5 \\
\hline 12 & $24 / 05 / 96$ & Barra do Descoberto - baixio vegetado com Crinum sp. & Manual & - \\
\hline 13 & $24 / 05 / 96$ & $\begin{array}{l}\text { Barra do Descoberto - baixio vegetado com Spartina } \\
\text { alterniflora }\end{array}$ & Manual & - \\
\hline 14 & $24 / 05 / 96$ & Barra do Descoberto - baixio não vegetado & Manual & - \\
\hline 15 & $24 / 05 / 96$ & $\begin{array}{l}\text { Barra do Rio do Cedro - baixio vegetado com Spartina } \\
\text { alterniflora }\end{array}$ & Manual & - \\
\hline 16 & $24 / 05 / 96$ & Barra do Rio do Cedro - baixio não vegetado & Manual & - \\
\hline 17 & $24 / 05 / 96$ & $\begin{array}{l}\text { Barra do Boguaçú - baixio vegetado com Spartina } \\
\text { alterniflora }\end{array}$ & Manual & - \\
\hline 18 & $24 / 05 / 96$ & Barra do Boguaçú - baixio não vegetado & Manual & - \\
\hline 19 & $14 / 06 / 96$ & Mirim - desembocadura de córrego (zona urbana) & Manual & - \\
\hline 20 & $14 / 06 / 96$ & $\begin{array}{l}\text { Mirim - desembocadura de córrego sobre o baixio não } \\
\text { vegetado (zona urbana) }\end{array}$ & Manual & - \\
\hline 21 & $14 / 06 / 96$ & $\begin{array}{l}\text { Mirim - desembocadura de córrego - baixio vegetado } \\
\text { com Spartina alterniflora }\end{array}$ & Manual & - \\
\hline 22 & 28/06/96 & Poitas dos barcos de pesca-Caieiras & Van Veen & 5 \\
\hline 23 & $28 / 06 / 96$ & Cais do Milionário 2 - lado de Guaratuba & Van Veen & 10 \\
\hline 24 & $28 / 06 / 96$ & Cais do Milionário - lado de Caieiras & Van Veen & 8 \\
\hline 25 & $28 / 06 / 96$ & Barra da baía de Guaratuba & Van Veen & 8 \\
\hline 26 & $14 / 07 / 96$ & Ilha da Garcinha & Arrasto de porta & - \\
\hline 27 & $20 / 07 / 96$ & $\begin{array}{l}\text { Illha da Sepultura - baixio vegetado com Spartina } \\
\text { alterniflora }\end{array}$ & Manual & - \\
\hline 28 & $20 / 07 / 96$ & Praia das Caieiras & Manual & - \\
\hline 29 & $20 / 07 / 96$ & Sublitoral ao largo da barra da Baia de Guaratuba & Arrasto de porta & 5 \\
\hline
\end{tabular}

\section{RESULTADOS E DISCUSSÃO}

A tabela II apresenta a lista de espécies registradas durante o levantamento. Um total de 69 taxa foram identificados, registrando maior número de poliquetas e moluscos bivalves. Do total identificado, 14 taxa $(20,3 \%)$ foram encontradas exclusivamente nos habitats entre-marés vegetados por Spartina alterniflora e/ou Clinum sp., 2 taxa (2,9\%) nos baixios entre-marés não vegetados, 27 taxa nos fundos sublitorais e $5 \operatorname{taxa}(7,2 \%)$ na praia arenosa. Do total de taxa listados no presente trabalho, apenas $22(31,9 \%)$ já haviam sido registrados para a área.

A lista de espécies da macrofauna bentônica da Baía de Guaratuba apresentada é preliminar, em virtude do curto período de tempo de amostragem no campo. Estudos quantitativos demandariam no mínimo uma série anual de amostragens. No litoral paranaense ocorrem um inverno e verão bem marcados, que geram os padrões de variação dos parâmetros ambientais, especialmente a temperatura. O regime de chuvas também é diferenciando, e os maiores índices de pluviosidade nos meses quentes pode causar diminuição da salinidade com eventuais conseqüências sobre as comunidades biológicas marinhas pelágicas e bentônicas. 
Tabela II. Espécies da Macrofauna Bentônica coletadas no presente levantamento, realizado na Baia de Guaratuba, Paraná. Os taxa ainda não citados para a Baía de Guaratuba encontram-se assinalados com um X. Habitats de ocorrência: (V) entre-marés vegetado, (NV) entre-marés não vegetado, (S) fundos sublitorais, $(P)$ praias arenosas.

\begin{tabular}{|c|c|c|c|}
\hline Taxa & Primeira citação & Estações de coleta & Habitat de ocorrência \\
\hline \multicolumn{4}{|l|}{ Polychaeta } \\
\hline \multicolumn{4}{|l|}{ Orbiniidae } \\
\hline Scoloplos sp. & $x$ & $2,5,17,18$ & V, NV, S \\
\hline \multicolumn{4}{|l|}{ Spionidae } \\
\hline Spiophanes missionensis Hartman, 1941 & $\mathrm{x}$ & 22 & S \\
\hline Paraprionospio pinnata (Ehlers, 1901) & $x$ & 22 & $\mathrm{~S}$ \\
\hline Polydora websteri Hartman, 1943 & $x$ & 27 & V \\
\hline Scolelepis squamata (Müller, 1806) & $x$ & 28 & $P$ \\
\hline \multicolumn{4}{|l|}{ Magelonidae } \\
\hline Magelona variolamellata Bolivar \& Lana, 1986 & $x$ & 3,4 & $\mathrm{~S}$ \\
\hline \multicolumn{4}{|l|}{ Capitellidae } \\
\hline Capitella capitata (Fabricius, 1780) & $x$ & $2,17,18,27$ & V, NV, S \\
\hline Heteromastus similis Southern, 1821 & $x$ & $8,9,10,14,27$ & V, NV, S \\
\hline \multicolumn{4}{|l|}{ Opheliidae } \\
\hline Euzonus furciferus (Ehlers, 1897) & $x$ & 28 & $\mathrm{P}$ \\
\hline Armandia sp. & $x$ & 23 & $\mathrm{~S}$ \\
\hline \multicolumn{4}{|l|}{ Pilargidae } \\
\hline Parandalia americana (Hartman, 1947) & & $4,5,11,17,22$ & V.S \\
\hline \multicolumn{4}{|l|}{ Nereididae } \\
\hline Neanthes succinea (Frey \& Leuckart, 1847) & $\mathrm{x}$ & $1,13,17,27$ & V, S \\
\hline Perinereis vancaurica (Ehlers, 1868) & $x$ & 6 & S \\
\hline Laeonereis acuta (Treadwell, 1923) & & $6,13,17,18,20$ & $V, N V, S$ \\
\hline Nereis oligohalina (Rioja, 1946) & $x$ & 27 & v \\
\hline \multicolumn{4}{|l|}{ Glyceridae } \\
\hline Hemipodus olivieri Orensanz \& Gianuca, 1974 & $x$ & 1 & $\mathrm{~S}$ \\
\hline \multicolumn{4}{|l|}{ Goniadidae } \\
\hline Goniada litorea Hartman, 1950 & $x$ & 3,22 & $\mathrm{~S}$ \\
\hline Goniada maculata Oersted, 1843 & $x$ & 3 & $\mathrm{~S}$ \\
\hline Glycinde multidens Müller, 1858 & & $5,11,17,20,21,22$ & V, NV, S \\
\hline \multicolumn{4}{|l|}{ Nephthyidae } \\
\hline Nephtys fluviatilis Monro, 1937 & & $6,7,8,9,13,14,17,18,21$ & V, NV, S \\
\hline \multicolumn{4}{|l|}{ Lumbrineridae } \\
\hline Lumbrineris sp. & $x$ & 27 & V \\
\hline \multicolumn{4}{|l|}{ Ampharetidae } \\
\hline Isolda pulchella Müller, 1858 & $x$ & $9,17,20,21,27$ & V.NV,S \\
\hline Sipuncula & $x$ & $2,3,5,11,18,21$ & V, NV, S \\
\hline \multicolumn{4}{|l|}{ Crustacea } \\
\hline \multicolumn{4}{|l|}{ Cirripedia } \\
\hline Balanidae & & 12 & $\mathrm{~V}$ \\
\hline \multicolumn{4}{|l|}{ Decapoda } \\
\hline Penaeidae & & & \\
\hline Litopenaeus schmitiï Burkenroad, 1936 & $\mathrm{x}$ & 29 & $\mathrm{~s}$ \\
\hline Xiphopenaeus kroyeri (Heller, 1862) & & 29 & $\mathrm{~S}$ \\
\hline Alpheidae & & & \\
\hline Alpheus estuariensis Christofferson, 1984 & $x$ & 8 & s \\
\hline Alpheus thomasi Hendrix \& Gore, 1973 & $\mathrm{x}$ & 17 & V \\
\hline Paguroidea & & 26 & $\mathrm{~S}$ \\
\hline Hippidae & & & \\
\hline Emerita brasiliensis Schmitt, 1935 & $x$ & 28 & $P$ \\
\hline Magidae & & & \\
\hline Libinia sp. & & 26 & $\mathrm{~S}$ \\
\hline Xanthidae & & & \\
\hline Eurythium limosum (Say, 1818) & & $15,17,20$ & $\mathrm{~V}, \mathrm{NV}$ \\
\hline Hexapanopeus paulensis Rathbun, 1930 & & 1 & S \\
\hline Portunidae & & & \\
\hline Callinectes danae Smith, 1869 & & 26,29 & $\mathrm{~S}$ \\
\hline Callinectes larvatus Ordway, 1863 & & 20 & $\mathrm{~S}$ \\
\hline Callinectes sapidus Rathbun, 1897 & & 13,14 & $\mathrm{~S}$ \\
\hline Grapsidae & & & \\
\hline Metasesarma rubripes (Rathbun, 1897) & & 12 & V \\
\hline Ocypodidae & & & \\
\hline Uca uruguayensis Nobili, 1901 & & 12,19 & $\begin{array}{l}\text { V, NV } \\
\text { Cont }\end{array}$ \\
\hline
\end{tabular}


Tabela II. Continuação

\begin{tabular}{|c|c|c|c|}
\hline Taxa & Primeira citação & Estações de coleta & Habitat de ocorrência \\
\hline \multicolumn{4}{|l|}{ Tanaidacea } \\
\hline \multicolumn{4}{|l|}{ Kalliapseudidae } \\
\hline Kalliapseudes schubartii Mane-Garzón, 1949 & $\mathrm{x}$ & $13,14,18$ & V.NV \\
\hline Sinelobus stanfordi (Richardson, 1901) & $x$ & 12 & v \\
\hline \multicolumn{4}{|l|}{ Isopoda } \\
\hline \multicolumn{4}{|l|}{ Sphaeromatidae } \\
\hline Sphaeroma terebrans Bate, 1866 & $x$ & 12 & V \\
\hline Sphaeroma annandalei Stebbing. 1911 & $x$ & 1 & $S$ \\
\hline Pseudosphaeroma jakobii Loyola e Silva & & $12,15,17,27$ & V \\
\hline Pseudosphaeroma mourei Loyola e Silva, 1960 & $x$ & 27 & V \\
\hline Cassidinidea fluminensis (Mane-Garzon, 1944) & $x$ & 27 & v \\
\hline Cirolanidae & & 28 & $P$ \\
\hline \multicolumn{4}{|l|}{ Idotheidae } \\
\hline Zenobiana planicauda Benedict, 1889 & & 1 & S \\
\hline \multicolumn{4}{|l|}{ Amphipoda } \\
\hline Corophiidae & & $8,12,13,14,17,18$ & V, NV, S \\
\hline \multicolumn{4}{|l|}{ Gamaridae } \\
\hline Grandidierella sp. & & 2,15 & $\mathrm{~V}, \mathrm{~s}$ \\
\hline Maera sp. & & 15 & V \\
\hline Platyischnopidae & & 11 & $S$ \\
\hline \multicolumn{4}{|l|}{ Mollusca } \\
\hline Polyplacophora & & 5 & $S$ \\
\hline \multicolumn{4}{|l|}{ Gastropoda } \\
\hline \multicolumn{4}{|l|}{ Taididade } \\
\hline Stramonita haemastoma (Linnaeus, 1767) & & 1 & $\mathrm{~s}$ \\
\hline \multicolumn{4}{|l|}{ Bullidae } \\
\hline Bulla striata Bruguiere, 1792 & & 4 & S \\
\hline \multicolumn{4}{|l|}{ Neritidae } \\
\hline Neritina virginea (Linnaeus, 1758) & & $15,17,27$ & V \\
\hline Pulmonata-Stylomatophora & & 9 & $\mathrm{~S}$ \\
\hline \multicolumn{4}{|l|}{ Bivalvia } \\
\hline \multicolumn{4}{|l|}{ Solecurtidae } \\
\hline Tagelus divisus (Spengler, 1794) & & $1,2,21$ & $\mathrm{~V}, \mathrm{~S}$ \\
\hline Tagelus plebeius (Lightfoot, 1786) & & 19 & NV \\
\hline \multicolumn{4}{|l|}{ Tellinidae } \\
\hline Strigilla pisiformis (Linnaeus 1758) & & 23 & S \\
\hline Macoma constricta (Bruguiere, 1792) & & $3,17,18,20$ & V, NV, S \\
\hline Tellina sp. A & & $2,5,18,21$ & V, NV, S \\
\hline Tellina sp. B & & 24 & s \\
\hline \multicolumn{4}{|l|}{ Corbulidae } \\
\hline Corbula caribaea Orbigni, 1842 & & 3 & $\mathrm{~S}$ \\
\hline \multicolumn{4}{|l|}{ Mytillidae } \\
\hline Mytella guyanensis (Lamarck, 1819) & & $2,5,15,19$ & V, NV, S \\
\hline \multicolumn{4}{|l|}{ Veneridae } \\
\hline Anomalocardia brasiliana (Gmelin, 1791) & & 27 & V \\
\hline \multicolumn{4}{|l|}{ Donacidae } \\
\hline Donax hanleyanus Philippi, 1842 & & 28 & $P$ \\
\hline Phoronida & & $3,10,21$ & V, S \\
\hline \multicolumn{4}{|l|}{ Echinodermata } \\
\hline Mellitidae & & & \\
\hline Mellita quinquiesperforata (Leske, 1778) & & 29 & $\mathrm{~S}$ \\
\hline Cephalochordata & & & \\
\hline Branchiostomatidae & & & \\
\hline Branchiostoma sp. & & 3,22 & S \\
\hline
\end{tabular}

TOMMASI (1970) forneceu um dos primeiros levantamentos da fauna bentônica do complexo estuarino-lagunar de Cananéia, São Paulo, e também observou a dominância de poliquetas nas associações de fauna de fundos infralitorais, perfazendo mais de $50 \%$ das espécies identificadas. BENVENUTI et al. (1978) e CAPITOLI et al. (1978) forneceram estudos da ecologia estuarial da Lagoa dos Patos, Rio Grande do Sul, onde concluíram que as associações da fauna bentônica foram dominadas por moluscos, no caso a espécie Littoridina australis (Orbigny, 1835) 
com densidades populacionais extremamente elevadas em setores próximo das barras de acesso da Lagoa dos Patos. Os poliquetas estiveram constantes nas amostragens mas não foram dominantes.

Durante dois anos de levantamentos na Baía de Paranaguá, Paraná, LANA (1986b) registrou 130 taxa da fauna bentônica. Considerando as dimensões da Baía de Paranaguá, o autor dividiu aquele estuário em setores com padrões de sedimentação e hidrográficos distintos onde a fauna de fundos moles apresenta composição de espécies altamente variável.

A Baía de Guaratuba apresentou um número de espécies proporcionalmente elevado para um curto período de amostragem, o que mostra que estudos detalhados da variabilidade espaço-temporal da fauna desse estuário devem fornecer listas de espécies ainda mais ricas. O conhecimento mais aprofundado sobre o papel ecológico destes sistemas estuarinos na zona costeira e os reflexos sobre a economia, podem contribuir para o desenvolvimento regional.

\section{REFERÊNCIAS BIBLIOGRÁFICAS}

Bemvenuti, C.E.; R.R. Capitoli \& N.M. Gianuca. 1978. Estudos de Ecologia bentônica na região estuarial da Lagoa das Patos. II - Distribuição quantitativa do macrobentos infralitoral. Atlântica, Rio Grande, 3: 23-32.

Bemvenuti, C.E.; S.A. Cattaneo \& S.A. Netto. 1992. Structural characteristics of the benthic macrofauna on two locations in the estuarine region of Patos Lagoon, RS, Brazil. Atlântica, Rio Grande, 14: 23-32

Bigarella, J.J. 1946. Contribuição ao estudo da Planície Litorânea do Estado do Paraná. Arq. Biol. Tecnol., Curitiba, 1: 75-111.

1978. A Serra do Mar e a Porção Oriental do Estado do Paraná. Governo do Paraná, Secretaria de Estado do Planejamento, ADEA, 249p.

Bjornberg, T.K.S. 1953. On a Balanoglossus from the coast of Paraná, Brasil. Dusenia, Curitiba, 4: 127-172.

CAPtToli, R.R.; C.E. Bemvenuti \& N.M. GianuCA. 1978. Estudos de Ecologia bentônica na região estuarial da Lagoa dos Patos. I-Comunidades bentônicas. Atlântica, Rio Grande, 3: 5-22.

GoFFERJÉ, C.M. 1950. Contribuição à zoogeografia da malacofauna do litoral do Estado do Paraná. Arq. do Museu Paranaense, Curitiba, 8: 221-294.

JAKoBI, H. 1953. Sobre a distribuição da salinidade e do pH na Baía de Guaratuba. Arq. do Museu Paranaense, Curitiba, 10: 3-35.

- 1955. O gênero Enhydrosoma no manguezal da costa São Paulo- Paraná (Harpacticoidea Crustacea). Dusenia, Curitiba, 6: 89-96.

LANA, P.C. 1986a. Nephtyidae (Annelida: Polychaeta) do litoral do Estado do Paraná (Brasil). Nerítica, Pontal do Sul, 1 (3): 79-89.

1986b. Macrofauna bêntica de fundos sublitorais não consolidados da Baía de paranaguá (Paraná). Nerítica, Pontal do Sul, 1 (3): 79-89.

Loyola E SiLVA, J. DE. 1959. Pseudosphaeroma jakobii n. sp. (Isopoda: Crustacea), encontrado na Baía de Guaratuba (PR - Brasil). Dusenia, Curitiba, 8: 79-88.

- 1992. Callapa sulcata Rathbun (Crustacea, Decapoda, Callapidae) de Guaratuba, Paraná, Brasil. Revta bras. Zool., Curitiba, 9 (1/2): 47-51.

MAACK, R. 1968. Geografia Física do Estado do Paraná. Curitiba, Livraria José Olímpio Ed., SCE-PR, $450 \mathrm{p}$.

Marcus, E. 1941. Briozoários marinhos do litoral paranaense. Arq. Mus. Paranaense, Curitiba, 1: 7-37.

Melo, G.A.S.; V.G. Veloso \& M.C. Oliveira. 1989. A fauna de Brachyura (Crustacea-Decapoda) do litoral do Estado do Paraná: lista preliminar. Nerítica, Pontal do Sul, 4: 1-31. 
Morretes, F.L. 1949. Ensaio de Catálogo dos Moluscos do Brasil. Arq. Mus. Paranaense, Curitiba, 7: 5-216.

Müller, A.C.P. \& P.C. LANa. 1986. Teredinidae (Mollusca: Bivalvia) do litoral do Paraná, Brasil. Nerítica, Pontal do Sul, 1:27-48.

Petrobrás. 1994. Diagnóstico ambiental oceânico e costeiro das regiões sul e sudeste do Brasil. Curitiba, Editora UFPR, Vol. 4, 200p.

SaWAyA, M.P. 1945. Anoplodactylus stictus Marc. (Pantopoda) em Caiobá, Estado do Paraná. Arq. Mus. Paranaense, Curitiba, 4: 231-234.

Tommasi, L.R. 1967. Observações preliminares sobre a fauna bêntica de sedimentos moles da Baía de Santos e regiões vizinhas. Bol. Inst. Oceanogr., São Paulo, 16 (1): 43-65.

. 1970. Observações sobre a fauna bêntica do complexo estuarino-lagunar de Cananéia, São Paulo, Bol. Inst. Oceanogr., São Paulo, 19: 43-65.

Recebido em 20.XII.2000; aceito em 23.VII.2002. 